B. A. Bhayo, J. SÁNdor

\title{
INEQUALITIES CONNECTING GENERALIZED TRIGONOMETRIC FUNCTIONS WITH THEIR INVERSES
}

\begin{abstract}
Motivated by the recent work [1], in this paper we study the relations of generalized trigonometric and hyperbolic functions of two parameters with their inverse functions.
\end{abstract}

Key words: Inequalities, generalized trigonometric functions, Eigenfunctions and Incomplete beta function.

2010 Mathematical Subject Classification: 33C99, 33B99.

\section{$\S 1$. Introduction}

In [2] P. Lindqvist studied generalized trigonometric and hyperbolic functions ( $p$-functions) for a parameter $p>1$, and for $p=2$ they coincide with elementary functions. These $p$-functions were studied extensively, see for example [2-9] and their references. Recently these functions have been extended to $(p, q)$-functions with two parameters $p, q>1$ in $[10-$ 13]. These functions coincide with the $p$-functions for $p=q$. For the historical background see the bibliography of these papers. In [14] and [1] authors have studied the inequalities involving elementary functions and their inverses. Thereafter in [14] Klén et al. studied those results in terms of $p$-functions. Here we generalized those inequalities for $(p, q)$ functions and establish double inequality for $\sin _{p}$ in terms of elementary functions, $\sin _{p}$ occurs as an eigenfunction of the Dirichlet problem for the one-dimensional $p$-Laplacian, see [6].

Before we formulate our main results we define the $(p, q)$-functions and some other notation. The increasing homeomorphism function $F_{p, q}$ : $[0,1] \rightarrow\left[0, \pi_{p, q} / 2\right]$ is defined by

$$
\arcsin _{p, q}(x)=\int_{0}^{x}\left(1-t^{q}\right)^{-1 / p} d t .
$$

(C) Bhayo B. A., Sándor J., 2013 
Letting $t=z^{1 / q}$, we have

$$
\arcsin _{p, q}(x)=\frac{1}{q} \int_{0}^{x^{q}} z^{1 / q-1}(1-z)^{-1 / p} d z=\frac{1}{q} \tilde{B}\left(\frac{1}{q}, 1-\frac{1}{p}, x^{q}\right),
$$

where $\tilde{B}(a, b, x)$ is incomplete beta function defined as

$$
\tilde{B}(a, b, x)=\int_{0}^{x} t^{a-1}(1-t)^{b-1} d t .
$$

The inverse of $\arcsin _{p, q}$ is denoted by $\sin _{p, q}$, which is defined on the interval $\left[0, \pi_{p, q} / 2\right]$, where

$$
\pi_{p, q}=2 \arcsin _{p, q}(1)=\frac{2}{q} \tilde{B}\left(\frac{1}{q}, 1-\frac{1}{p}, 1\right)=\frac{2}{q} B\left(\frac{1}{q}, 1-\frac{1}{p}\right),
$$

here $B(a, b)$ denote the beta function. We also define

$$
\arccos _{p, q} x=\arcsin _{p, q}\left(\left(1-x^{p}\right)^{1 / q}\right)
$$

(see [11, Prop. 3.1]), and

$$
\cos _{p, q}(x)=\frac{d}{d x} \sin _{p, q}(x), \quad x \in\left[0, \pi_{p, q} / 2\right] .
$$

Letting $y=\sin _{p, q}(x)$, we get

$$
\cos _{p, q}(x)=\left(1-\left(\sin _{p, q}(x)\right)^{q}\right)^{1 / p},
$$

and

$$
\left|\cos _{p, q}(x)\right|^{p}+\left|\sin _{p, q}(x)\right|^{q}=1 .
$$

The generalized tangent function $\tan _{p, q}(x)$ is defined as

$$
\tan _{p, q}(x)=\frac{\sin _{p, q}(x)}{\cos _{p, q}(x)}
$$

For $x \in(0, \infty)$, the inverse of generalized hyperbolic sine function $\sinh _{p, q}(x)$ is defined by

$$
\operatorname{arcsinh}_{p, q} x=\int_{0}^{x}\left(1+t^{q}\right)^{-1 / p} d t
$$


and generalized hyperbolic cosine and tangent functions are defined by

$$
\cosh _{p, q}(x)=\frac{d}{d x} \sinh _{p, q}(x), \quad \tanh _{p, q}(x)=\frac{\sinh _{p, q}(x)}{\cosh _{p, q}(x)}, \quad x \geq 0
$$

respectively. It follows from the definitions, that

$$
\left|\cosh _{p, q}(x)\right|^{p}-\left|\sinh _{p, q}(x)\right|^{q}=1, \quad x \geq 0 .
$$

The main results of the this paper reads as below.

Theorem 1. For $p, q>1$ the following hold

1) For all $x \in(0,1)$ and $y \in\left(0, \pi_{p, q} / 2\right)$ with $y<\arcsin _{p, q}(x)$ we have

$$
\arcsin _{p, q}(x) \sin _{p, q}(y)>x y \text {. }
$$

2) For all $x \in\left(0, \pi_{p, q} / 2\right)$ and $y \in(0,1)$ with $\tan _{p, q}(x)>y$ we have

$$
\tan _{p, q}(x) \arctan _{p, q}(y)>x y .
$$

3) For all $x, y \in(0, \infty)$ with $y<\sinh _{p, q}(x)$ we have

$$
\sinh _{p, q}(x) \operatorname{arcsinh}_{p, q}(y)>x y .
$$

4) For all $x \in(0,1)$ and $y \in(0, \infty)$ with $\operatorname{arctah}_{p, q}(x)>y$ we have

$$
\operatorname{arctah}_{p, q}(x) \tanh _{p, q}(y)>x y .
$$

Theorem 2. For $p, q>1$ the following hold

1) $\frac{x}{\arcsin _{p, q}(x)}>\frac{\sin _{p, q}\left(\pi_{p, q} x / 2\right)}{\pi_{p, q} x / 2}, \quad x \in(0,1)$,

2) $\frac{\tan _{p, q}(x)}{x}<\frac{b x}{\arctan _{p, q}(b x)}, \quad x \in(0, k), 0<k<\frac{\pi_{p, q}}{2}$, $b=\tan _{p, q}(k) / k$,

3) $\frac{\sinh _{p, q}(x)}{x}<\frac{x}{a \arctan _{p, q}(x / a)}, \quad x \in(0, k), k>0, a=\frac{k}{\sinh _{p, q}(k)}$.

4) $\frac{x}{\operatorname{arctanh}_{p, q}(x)}>\frac{\tanh _{p, q}(c x)}{c x}, \quad x \in(0, k), k \in(0,1)$, $c=k / \operatorname{arctanh}_{p, q}(k)$. 


\section{$\S 2$. Preliminaries and proofs}

The following derivative formulas will be used in our calculations, and they can be derived easily from the definition.

Lemma 1. For all $x \in\left(0, \pi_{p, q} / 2\right)$, we have

1) $\frac{d}{d x} \cos _{p, q}(x)=-\frac{p}{q}\left(\cos _{p, q}(x)\right)^{2-p}\left(\sin _{p, q}(x)\right)^{q-1}$

2) $\frac{d}{d x} \tan _{p, q}(x)=1+\frac{p}{q} \frac{\left(\sin _{p, q}(x)\right)^{q}}{\left(\cos _{p, q}(x)\right)^{p}}$,

and for all $x \in(0, \infty)$

3) $\frac{d}{d x} \cosh _{p, q}(x)=\frac{q}{p}\left(\cosh _{p, q}(x)\right)^{2-p}\left(\sinh _{p, q}(x)\right)^{q-1}$,

4) $\frac{d}{d x} \tanh _{p, q}(x)=1-\frac{q}{p} \frac{\left(\sinh _{p, q}(x)\right)^{q}}{\left(\cosh _{p, q}(x)\right)^{p}}$.

For the following monotone l'Hospital rule see [15, Theorem 1.25].

Lemma 2. For $-\infty<a<b<\infty$, let $f, g:[a, b] \rightarrow \mathbb{R}$ be continuous on $[a, b]$, and be differentiable on $(a, b)$. Let $g^{\prime}(x) \neq 0$ on $(a, b)$. If $f^{\prime}(x) / g^{\prime}(x)$ is increasing (decreasing) on $(a, b)$, then so are

$$
\frac{f(x)-f(a)}{g(x)-g(a)} \text { and } \quad \frac{f(x)-f(b)}{g(x)-g(b)} .
$$

If $f^{\prime}(x) / g^{\prime}(x)$ is strictly monotone, then the monotonicity in the conclusion is also strict.

For the proof of following lemma see $([1])$.

Lemma 3. Let $f: I \rightarrow J$ be a injective function, where $I, J$ are the subsets of $(0, \infty)$. Suppose that the function $g(x)=f(x) / x, x \in I$ is strictly increasing. Then for any $x \in I, y \in J$ such that $f(x) \geq y$ following holds

$$
f(x) f^{-1}(y) \geq x y,
$$

where $f^{-1}: J \rightarrow I$ denotes the inverse function of $f$. Under the same condition if $f(x) \leq y$ then we have

$$
f(x) f^{-1}(y) \leq x y
$$


For the following lemma see [16, Theorem 2, p. 151], [13, Theorem 1].

\section{Lemma 4.}

1) Let $J \subset \mathbb{R}$ be an open interval, and $f: J \rightarrow \mathbb{R}$ be a strictly monotonic function. Let $f^{-1}: f(J) \rightarrow J$ be the inverse of $f$. If $f$ is concave and increasing, then $f^{-1}$ is convex.

2) For all $x \in(0,1)$, the functions $p \mapsto \arcsin _{p}(x)$ and $p \mapsto \operatorname{arctanh}_{p}(x)$ are strictly decreasing in $p \in(1, \infty)$.

Lemma 5. For $p, q>1$, the following hold

1) the function $f(x)=\frac{\arcsin _{p, q}(x)}{x}$ is increasing in $x \in(0,1)$,

2) the function $g(x)=\frac{\tan _{p, q}(x)}{x}$ is increasing in $x \in\left(0, \pi_{p, q} / 2\right)$,

3) the function $h(x)=\frac{\sinh _{p, q}(x)}{x}$ is increasing in $x \in(0, \infty)$,

4) the function $j(x)=\frac{\operatorname{arctah}_{p, q}(x)}{x}$ is increasing in $x \in(0, \infty)$ with $p>q$.

Proof. Let $f(x)=\frac{\arcsin _{p, q}(x)}{x}=\frac{f_{1}(x)}{f_{2}(x)}$. Then $f_{1}^{\prime}(x)=\left(1-x^{q}\right)^{-1 / p}>0$ and $f_{2}^{\prime}(x)>0$. Now it is clear by Lemma 2 that $f$ is increasing. For the proof of part (2) and (3), let

$$
g(x)=\frac{\tan _{p, q}(x)}{x}=\frac{g_{1}(x)}{g_{2}(x)}, h(x)=\frac{\sinh _{p, q}(x)}{x}=\frac{h_{1}(x)}{h_{2}(x)} .
$$

Differentiation gives

$$
g_{1}^{\prime}(x)=1+\frac{p}{q} \frac{\left(\sin _{p, q}(x)\right)^{q}}{\left(\cos _{p, q}(x)\right)^{p}}>0, \quad \text { and } \quad h_{1}^{\prime}(x)=\cosh _{p, q}(x)>0
$$

and the proof is obvious from Lemma 2. For part (4), we get

$$
\begin{aligned}
\frac{d^{2}}{d x^{2}} \tanh _{p, q}(x) & =-\frac{q}{p}\left(\frac{q\left(\sinh _{p, q}(x)\right)^{q-1}\left(\cosh _{p, q}(x)\right)^{p+1}-q \cosh _{p, q}(x)}{\left(\sinh _{p, q}(x)\right)^{2 q-1}}\right)= \\
& =-\frac{q}{p}\left(\sinh _{p, q}(x)\right)^{q-1}\left(\cosh _{p, q}(x)\right)^{1-2 p}<0,
\end{aligned}
$$


since $\tanh _{p, q}(x)$ is concave, and clearly with $p>q$ it is increasing. By Lemma $4(1), \operatorname{arctah}_{p, q}(x)$ is convex, and from this fact we get, that

$$
\frac{d}{d x} \operatorname{arctah}_{p, q}(x)
$$

is increasing. Hence the rest of proof follows from Lemma 2 .

Proof of Theorem 1. The functions

$$
\frac{\arcsin _{p, q}(x)}{x}, \quad \frac{\tan _{p, q}(x)}{x} \quad \frac{\sinh _{p, q}(x)}{x}, \text { and } \frac{\operatorname{arctah}_{p, q}(x)}{x}
$$

are increasing by Lemma 5 . The rest of proof follows immediately from Lemma 3.

It is easy to check by using the derivative formulas that the following relations

$$
\begin{gathered}
x<\arcsin _{p, q}(x), \quad x \in(0,1), \\
x<\tan _{p, q}(x), \quad x \in\left(0, \pi_{p, q} / 2\right), \\
x<\sinh _{p, q}(x), \quad x \in(0, \infty), \\
x>\tanh _{p, q}(x) \Rightarrow \operatorname{arctanh}_{p, q}(x)>x, \quad x \in(0,1) .
\end{gathered}
$$

hold true for all $p, q>1$.

By Theorem 1 and above relations we conclude the following corollary.

Corollary. For $p, q>1$ the following hold

1) $\frac{x}{\arcsin _{p, q}(x)}<\frac{\sin _{p, q}(x)}{x}, \quad x \in(0,1)$,

2) $\frac{x}{\arctan _{p, q}(x)}<\frac{\tan _{p, q}(x)}{x}, \quad x \in(0,1)$,

3) $\frac{x}{\operatorname{arcsinh}_{p, q}(x)}<\frac{\sinh _{p, q}(x)}{x}, \quad x \in(0, \infty)$,

4) $\frac{x}{\operatorname{arctanh}_{p, q}(x)}<\frac{\tanh _{p, q}(x)}{x}, \quad x \in(0,1)$. 
Proof of Theorem 2. The monotonicity of the functions

$$
\frac{\arcsin _{p, q}(x)}{x}, \quad \frac{\tan _{p, q}(x)}{x} \quad \frac{\sinh _{p, q}(x)}{x}, \quad \frac{\operatorname{arctah}_{p, q}(x)}{x}
$$

imply, that

$$
\begin{gathered}
f_{1}(x)=\frac{\pi_{p, q}}{2} \arcsin _{p, q}(x)<x, \\
f_{2}(x)=\frac{\tan _{p, q}(x)}{b}<x, \\
f_{3}(x)=a \sinh _{p, q}(x)<x, \\
\text { and } f_{4}(x)=\operatorname{carctanh}_{p, q}(x)<x .
\end{gathered}
$$

Hence

$$
\begin{gathered}
f_{1}^{-1}(x)=\sin _{p, q}\left(\pi_{p, q} x / 2\right), \quad f_{2}^{-1}(x)=\arctan _{p, q}(b x), \\
f_{3}^{-1}(x)=\operatorname{arcsinh}_{p, q}(x / a), \quad f_{4}^{-1}(x)=\operatorname{arctanh}_{p, q}(c x),
\end{gathered}
$$

and the proof follows from (3) if we let $y=x$.

Corollary. The following assertions hold true:

1) $\frac{x}{\arcsin (x)}<\frac{\sin _{p}(x)}{x}, \quad$ for $x \in(0,1), p \geq 2$,

2) $\frac{\sin _{p}(x)}{x}<\frac{2 x / \pi_{p}}{\arcsin \left(2 x / \pi_{p}\right)}$, for $x \in\left(0, \pi_{2}\right), p \in(1,2]$,

3) $\frac{x}{\arctan (x)}<\frac{\tan _{p}(x)}{x}, \quad$ for $x \in(0,1), p \in(1,2]$,

4) $\frac{\tan _{p}(x)}{x}<\frac{b x}{\arctan (b x)}$, for $x \in(0, k), 0<k<\pi_{p} / 2, b=\frac{\tan (k)}{k}$.

The proof follows from Theorem 1, Lemma 4(2) and Corollary 2.

Remark. In [17, Theorem 2.3], the following inequalities was proved

$$
\tilde{B}(a, b, x) \tilde{B}(a, b, y) \leq \tilde{B}(a, b, x+y-z) \tilde{B}(a, b, z)
$$

for $a \in(0,1), b>0$ and $x, y>z$. Under the same assumption with $0<x+y-z<1$ and $x, y, z \in(0,1)$ one has

$$
\arcsin _{p, q}(x) \arcsin _{p, q}(y) \leq \arcsin _{p, q}(x+y-z) \arcsin _{p, q}(z) .
$$




\section{References}

[1] Sándor J. On certain inequalities for hyperbolic and trigonometric functions, J. Math. Ineq. http://files.ele-math.com/preprints/jmi-1062-pre.pdf.

[2] Lindqvist P. Some remarkable sine and cosine functions. Ricerche di Matematica, Vol. XLIV (1995), 269-290.

[3] Bhayo B. A., Vuorinen M. Inequalities for eigenfunctions of the p-Laplacian. January 2011, 23 pp. arXiv math.CA 1101.3911.

[4] Bushell P. J., Edmunds D. E. Remarks on generalised trigonometric functions. Rocky Mountain J. Math. 42 (2012), Number 1, 25-57.

[5] Biezuner R. J., Ercole G., Martins E. M. Computing the first eigenvalue of the p-Laplacian via the inverse power method. J. Funct. Anal. 257 (2009), no. 1, 243-270.

[6] Drábek P., Manásevich R. On the closed solution to some p-Laplacian nonhomogeneous eigenvalue problems. Differential Integral Equations 12 (1999), no. 6, 773-788.

[7] Klén R., Vuorinen M., Zhang X. Inequalities for the generalized trigonometric and hyperbolic functions. October 2012, 12 pp. arXiv:1210.6749.

[8] Lang J., Edmunds D. E. Eigenvalues, Embeddings and Generalised Trigonometric Functions. Lecture Notes in Mathematics 2016, Springer-Verlag, 2011.

[9] Lindqvist P., Peetre J. p-arclength of the q-circle. The Mathematics Student, Vol. 72, (2003), Nos. 1-4, 139-145.

[10] Bhayo B. A., Vuorinen M. On generalized trigonometric functions with two parameters. J. Approx. Theory 164 (2012), 1415-1426.

[11] Edmunds D. E., Gurka P., Lang J. Properties of generalized trigonometric functions. J. Approx. Theory 164 (2012) 47-56, doi:10.1016/j.jat.2011.09.004.

[12] Takeuchi S. Generalized Jacobian elliptic functions and their application to bifurcation problems associated with p-Laplacian. J. Math. Anal. Appl. 385 (2012), 24-35, doi:10.1016/j.jmaa.2011.06.063.

[13] Baricz Á. , Bhayo B. A., Vuorinen M. Turán type inequalities for generalized inverse trigonometric functions, May 2013, 10 pp., arXiv:1305.0938 [math.CA].

[14] Klén R., Visuri M., Vuorinen M. On Jordan type inequalities for hyperbolic functions, J. Ineq. Appl., vol. 2010, pp. 14.

[15] Anderson G. D., Vamanamurthy M. K., Vuorinen M. Conformal invariants, inequalities and quasiconformal maps. J. Wiley, 1997, 505 pp. 
[16] Kuczma M. An introduction to the theory of functional equations and inequalities. Cauchy's equation and Jensen's inequality. With a Polish summary. Prace Naukowe Uniwersytetu Ślaskiego w Katowicach [Scientific Publications of the University of Silesia], 489. Uniwersytet Ślaski, Katowice; Państwowe Wydawnictwo Naukowe (PWN), Warsaw, 1985. 523 pp. ISBN: 83-01-05508-1.

[17] Sroysang B. Inequalities for the incomplete beta function, Mat. Aeterna, 3(2013), no., 4, 241-244.

[18] Abramowitz M. and Stegun I., eds. Handbook of mathematical functions with formulas, graphs and mathematical tables. National Bureau of Standards, 1964 (Russian translation, Nauka 1979).

[19] Baricz Á. Functional inequalities involving special functions II. J. Math. Anal. Appl. 327 (2007), no. 2, 1202-1213.

[20] Carlson B. C. Some inequalities for hypergeometric functions. Proc. of Amer. Math. Soc., vol. 17, (1966), no. 1, 32-39.

[21] Y.-M Chu, Y.-P Jiang and M.-Kun Wang. Inequalities for generalized trigonometric and hyperbolic sine functions. December 2012, $7 \mathrm{pp}$. arXiv:1212.4681

[22] W.-D. Jiang and F. Qi. Geometric convexity of the generalized sine and the generalized hyperbolic sine, arXiv.1301.3264.

The work is received on September 2, 2013.

University of Jyväskylä,

Department of Mathematical Information Technology,

40014 Jyväskylä, Finland.

E-mail: bhayo.barkat@gmail.com

Babeş-Bolyai University, Department of Mathematics,

Str. Kogalniceanu nr. 1, 400084 Cluj-Napoca, Romania.

E-mail: jsandor@math.ubbcluj.ro 